\title{
The degree of arm elevation impacts the endurance and cardiopulmonary adaptations of COPD patients performing upper-limb exercise: a cross-over study
}

\author{
Mara PANERONI 1 *, Carla SIMONELLI 1, Pierantonio LAVENEZIANA 2, 3 , \\ Massimiliano GOBBO ${ }^{4}$, Manuela SALERI ${ }^{1}$, Luca BIANCHI ${ }^{1}$, Michele VITACCA ${ }^{1}$
}

1Istituti Clinici Maugeri, IRCCS, Pavia, Italy; 2Division of Experimental and Clinical Respiratory Neurophysiology, Sorbonne University, Paris, France; '3Pitié-Salpêtrière Charles Foix Hospital Group, Service des Explorations Fonctionnelles de la Respiration, de l'Exercice et de la Dyspnée (Département “R3S”, Pôle Pragues), Paris, France; "Department of Physiology, University of Brescia, Brescia, Italy

*Corresponding author: Mara Paneroni, Istituti Clinici Scientifici Maugeri, Via S. Maugeri 4, 27100 Pavia, Italy. E-mail: mara.paneroni@icsmaugeri.it

\section{A B S T R A C T}

BACKGROUND: In Chronic Obstructive Pulmonary Disease (COPD), upper limb exercise is widely recommended. However, how the degree of shoulder flexion may influence the exercise response is unknown.

AIM: We compared metabolic, ventilatory and symptomatic responses during constant-load supported and unsupported exercise performed at $80^{\circ}$ and $120^{\circ}$ arm elevation.

DESIGN: Randomized cross-over study.

SETTING: Pulmonary Pathophysiology Service in an Italian Respiratory Rehabilitative Division, in-patients were enrolled.

METHODS: Twelve patients with moderate-to-severe COPD ( $\mathrm{FEV}_{1} 51 \%$, BMI $\left.26.7 \pm 6.3 \mathrm{Kg} / \mathrm{m}^{2}\right)$ performed 4 symptom-limited constant-load tests at $70 \%$ of their individual maximal workload: 2 supported and 2 unsupported, respectively at $80^{\circ}$ and $120^{\circ}$ of glenohumeral joint flexion, executed in a random order.

RESULTS: Time to exhaustion $\left(\mathrm{T}_{\mathrm{lim}}\right)$, evaluated by Kaplan-Maier curve, was shorter at $120^{\circ}$ than $80^{\circ}$ arm elevation in both supported (360 vs.. 486 seconds, $\mathrm{p}=0.031)$ and unsupported exercise $(210$ vs.. 375 seconds, $\mathrm{p}=0.005)$. No difference in dynamic hyperinflation was found between $80^{\circ}$ and $120^{\circ}$ elevation, even at the peak of exercise and at iso-ventilation. When normalized to $\mathrm{T}_{\text {lim }}, 120^{\circ}$ arm elevation had a significantly higher metabolic cost, heart rate, minute ventilation and dyspnea/fatigue symptoms compared to $80^{\circ}$ elevation, both in unsupported and supported conditions.

CONCLUSIONS: A larger shoulder flexion shortens per se exercise endurance due to the increased metabolic, ventilatory and cardiac response, without worsening dynamic hyperinflation.

CLINICAL REHABILITATION IMPACT: Arm position should be considered when prescribing individual exercise training and may be adjusted to modulate the workload.

(Cite this article as: Paneroni M, Simonelli C, Laveneziana P, Gobbo M, Saleri M, Bianchi L, et al. The degree of arm elevation impacts the endurance and cardiopulmonary adaptations of COPD patients performing upper-limb exercise: a cross-over study. Eur J Phys Rehabil Med 2018;54:690-7. DOI: 10.23736/S1973-9087.18.04962-6)

KEY WORDS: Exercise - Exercise test - Physical therapy modalities - Arm.

Cing Thronic obstructive pulmonary disease (COPD) leads to dyspnea and, subsequently, to systemic dysfunctions such as effort intolerance and progressive deconditioning. ${ }^{1-4}$ Furthermore, exercise in COPD patients can be strongly limited by the development of dynamic hyperinflation (DH), ${ }^{5}$ which may be directly related to the indi- vidual ventilatory response $\mathrm{e}^{3,5}$ or to the workload imposed during the training program. ${ }^{6}$ In recent years, the role of lower and upper body exercise in promoting functional independence has been widely recognized as a fundamental component of rehabilitation for patients with COPD with high level of evidence ${ }^{7,8}$ However, there is little evidence 


\section{COPYRIGHT $^{(\odot)} 2018$ EDIZIONI MINERVA MEDICA}

available on arm training, in particular concerning the most effective intensity or the most appropriate exercise modality for each patient. ${ }^{9-12}$

In pulmonary rehabilitation, many exercise modalities are used for arm training. Two main subgroups can be distinguished: supported upper-limb exercise (typically applied through arm cranking) and unsupported upper-limb exercise (by moving the arms without external aid).

Only few physiological studies have tested different arm exercise modalities to identify which are the most appropriate interventions for COPD rehabilitation. ${ }^{13-15}$ They compared the responses obtained during supported and unsupported exercise showing that both can induce dynamic hyperinflation..$^{15}$

Nevertheless, since unsupported training appeared to be the only modality able to improve the metabolic cost of the characteristic unsupported arm activities during daily life, this modality has been recommended as the optimal approach for arm training. ${ }^{6}$

Arm training is usually performed with some degrees of shoulder flexion, which, in turn, is known to influence the ventilatory response both in healthy subjects ${ }^{16}$ and in patients with lung disease. ${ }^{16,17}$ Baarends et al. showed that static arm elevation, maintained for 2 minutes, can increase the metabolic and ventilatory response, especially in COPD patients..$^{18}$ To date, despite the fact that dynamic upper-limb exercises are widely used in rehabilitative settings, only one study ${ }^{19}$ describes that strength exercises - performed with the arm elevation above shoulder level - appears to be more challenging than low arm elevation exercises, but no study evaluated the influence of the degree of shoulder elevation on patients' performance during upper limbs endurance exercises. Moreover, as activities of the upper limbs are often essential to maintain independence in daily life, a huge evaluation of the burden related to the tasks needs to be understood. Determining physiological responses and symptoms of different arm activities could help us to understand the mechanisms behind the difficulties that people with COPD experience when performing upper limb activities, and could help to define how exercise training should be prescribed.

On these bases, the aim of this study was to evaluate the impact of arm elevation on the performance (time to exhaustion) and the physiological responses of patients with moderate-to-severe COPD during different constant-load, dynamic upper-limb exercises (supported vs.. unsupported), being our hypothesis that higher elevation degrees may worse the exercise tolerance and high the risk of dynamic hyperinflation.

\section{Materials and methods}

This is a prospective, crossover study with different interventions provided in random order. We included 12 patients referred at the Respiratory Rehabilitation Division of the Istituti Clinici Scientifici Maugeri, Institute of Lumezzane (Brescia), Italy, between May 2011 and September 2012. The Ethics Committee of the Institute approved the protocol (CEC n $\left.{ }^{\circ} 707,18 / 04 / 2011\right)$. Signed informed consent was obtained from all participants.

This study conforms to all STROBE guidelines and reports the required information accordingly (see Supplementary Checklist).

\section{Patients}

Patients who met the following criteria were eligible for the study: diagnosis of moderate-to-severe COPD based on the American Thoracic Society/European Respiratory Society criteria; 20 clinical stability (no exacerbations or medication change within the previous 30 days); no previous participation in a pulmonary rehabilitation program within the past year. COPD diagnosis and severity level were established by means of pulmonary function tests conducted before and after administration of bronchodilators. Exclusion criteria were: severe comorbidities such as cardiac, orthopedic or neurological diseases; extreme difficulty or inability to perform the exercise protocol; need for oxygen supplementation.

\section{Protocol}

\section{Preliminary phase}

At the first day, all patients performed two incremental tests (supported/unsupported) to define the maximal workload they could do in supported and unsupported exercises. A period of 5 hours was interposed between the tests.

The incremental supported test was performed with an arm-ergometer (Rehab Trainer 881E, Monark Exercise $\mathrm{AB}$, Vansbro, Sweden) placed on a table in front of the seated patient. The arm crank height was adjusted so that the fulcrum of the pedals was at the level of the glenohumeral joint $\left(90^{\circ}\right.$ shoulder elevation). Before commencing the protocol, patients were familiarized with the exercise equipment (arm-ergometer). After the basal parameters had been recorded, patients performed a 1-minute unloaded warm-up, followed by an incremental phase in which the load was increased by 5 watts at the end of each minute. Patients were asked to maintain the speed revolution between 50 and $60 \mathrm{rpm}$, and were encouraged to continue 


\section{COPYRIGHT $^{\circledR} 2018$ EDIZIONI MINERVA MEDICA}

exercising until exhaustion (due to dyspnea or fatigue). The operator stopped the test when the maximal theoretical heart rate (HR) was achieved, or if severe arrhythmias or desaturation occurred, i.e. oxygen saturation $\left(\mathrm{SpO}_{2}\right)<80 \%$.

For the incremental unsupported test we used the Takahashi test: the patient was sitting on a chair holding a stick with the hands. We asked the patients to move with regular frequency (40 movements/min) the stick from the chest to a fixed level of a billboard in front of them (flexion/ extension of the elbow), starting from the knee level and raising the stick $15 \mathrm{~cm}$ every minute. If patients reached the higher level on the billboard (level 8), patients continued the exercise at $8^{\text {th }}$ level increasing the weight of the stick (500g per minute). More details about the procedure of the test are provided elsewhere..$^{21}$ The test was stopped if: 1) the maximum theoretical HR was achieved (208bpm- 0.7 $\mathrm{X}$ age in years);22 or 2) severe arrhythmias or severe desaturation $\left(\mathrm{SpO}_{2}<80 \%\right)$ occurred; or 3$)$ patient exhaustion (due to dyspnea or fatigue) occurred, or d) the patient was unable to perform the movement correctly.

\section{Experimental phase}

After the preliminary functional evaluation, four constantload tests (supported/unsupported at $80 / 120^{\circ}$ shoulder elevation, respectively) within a period of 4 days were performed. Among each test a period of rest of at least 5 hours was taken.

\section{SUPPORTED TEST}

After 1 minute of unloaded warm-up, for both $80^{\circ}$ and $120^{\circ}$, we set the workload of the arm-ergometer at the equivalent of $70 \%$ of each patient's maximal workload obtained during the incremental test.

\section{UNSUPPORTED TESTS}

Patients were asked to move the stick from the chest to a line on the billboard corresponding to $80^{\circ}$ and $120^{\circ}$ of flexion of the glenohumeral joint. For both conditions, after 1 min of unloaded warm-up, we loaded the stick at the equivalent of $70 \%$ of the maximal workload obtained by each patient in the incremental test and we asked the patient to maintain the effort as long as possible. For all constant-load tests, the time-to-exhaustion $\left(\mathrm{T}_{\mathrm{lim}}\right)$ was defined in any of the following conditions: the maximum HR (preliminary calculated through the incremental test) was achieved; severe arrhythmias or desaturation $\left(\mathrm{SpO}_{2}<80 \%\right)$ occurred; the patients perceived severe fatigue (BORG scale for dyspnea and muscular fatigue higher than 8).23

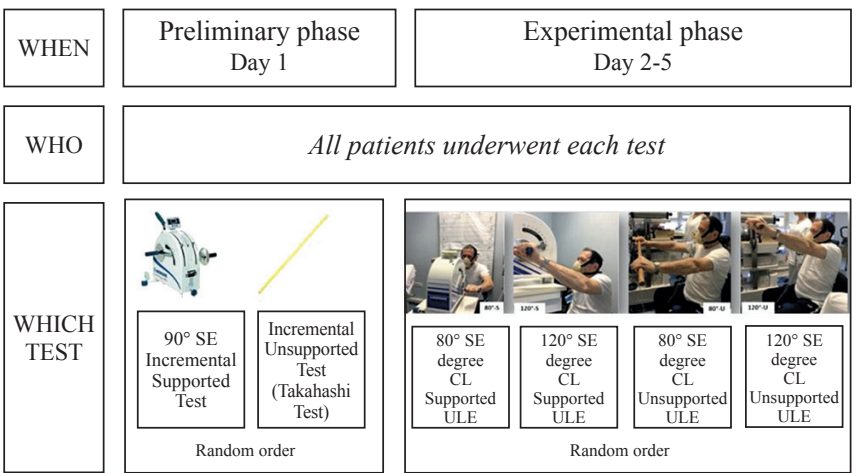

Figure 1.- Representative images of study design.

SE: shoulder elevation; CL: constant-load; ULE: upper limb exercise.

Figure 1 shows the study design and the four experimental conditions. The order of the two incremental tests during the preliminary phase and of the 4 constant-load tests during the experimental phase were randomized using an online dedicated software (http://www.randomization.com).

\section{Measurements}

During all tests, the measurements were carried out by means of a breath-by-breath analyzer (Vmax series 29c; Sensormedics, Milan, Italy). The tests were performed with a mouthpiece or mask, depending on the patient's preference. Electrocardiogram (ECG) monitoring was carried out during the tests through a system of electrodes connected to a 6x2-lead electrocardiograph (Mortara Instruments, Milan, Italy). The peripheral oxygen saturation was measured by a pulse-oximeter placed on a finger (8500A; Nonin Medical, Plymouth, MN, USA. The primary outcome of the study was the $\mathrm{T}_{\mathrm{lim}}$ for each test.

The variables evaluated and collected continuously during every test were the following:

1) Ventilatory variables: minute ventilation (VE), tidal volume $(\mathrm{Vt})$, respiratory rate $(\mathrm{RR})$ and inspiratory capacity (IC). IC measurement was conducted as follows: when a stable end-expiratory volume was reached, the patients were asked to take a deep breath up to total lung capacity (TLC). To give reproducible results, a minimum of three maneuvers at the start of every test was performed. IC value was obtained by subtracting the end-expiratory lung volume to TLC. 24,25 This measure was also performed each minute during the constant-load tests.

2) Cardiac parameter: HR.

3) Metabolic parameters: oxygen consumption $\left(\mathrm{VO}_{2}\right)$; carbon dioxide production $\left(\mathrm{VCO}_{2}\right)$; respiratory quotient (RQ). 


\section{COPYRIGHT $^{\odot} 2018$ EDIZIONI MINERVA MEDICA}

4) Symptom modifications: a modified Borg scale ${ }^{23}$ from 0 (null) to 10 (maximal score) was used to assess the patients' intensity of dyspnea and rate of perceived exertion.

For the study, we analyzed the differences in the exercise response in different ways.

i. at the end of exercise (peak).

ii. Normalizing each variable to the time of exercise $\left(T_{\text {lim }}\right)$. We calculated the range of change of each variable over $\mathrm{T}_{\lim }$ (in seconds) dividing the peak exercise - baseline difference by the exercise time. This allowed us to detect changes of each variable / second.

iii. At iso-VE, i.e. at the maximum level of $\mathrm{VE}$ reached by each patient in each test.

\section{Statistical analysis}

Data analysis was performed with a dedicated statistical software (StataCorp. LP; Stata Statistical Software: Release 12, 2011; College Station, TX, USA) by a blinded researcher not involved in the study protocol. In the descriptive analysis, continuous variables were expressed as means \pm standard deviation (SD) and binary ones as percentages. A sample size of 12 subjects was calculated considering a difference in performance between $120^{\circ}$ and $80^{\circ}$ tests of $75 \pm 80 \mathrm{sec}-$ onds, with alpha error $=0.05$ and beta error $=0.90$, according to the previous 5 tests conducted to test the feasibility of the protocol (no references in the literature were found). Based on the variability of the IC measurement reported in different studies, $, 6,21,24$ to detect differences in the $\mathrm{T}_{\text {lim }}$ between $120^{\circ}$ and $80^{\circ}$ elevation in the supported and unsupported exercises, a descriptive analysis using Kaplan-Meier curves and Log-Rank test was conducted. To detect differences in cardiorespiratory and metabolic parameters and in symptoms, we performed a non-parametric analysis using the paired-sample Wilcoxon signed-rank test. Statistical significance was considered for alpha $<0.05$.

\section{Results}

Patients' demographic and functional respiratory characteristics are shown in Table I.

Patients $(\mathrm{N} .=12)$ were predominantly male, elderly, with normal-weight and with a moderately decreased forced expiratory volume at 1 second $\left(\mathrm{FEV}_{1}\right)$ and a severely increased residual volume (RV). No abnormality in the gas exchange parameters was detected.

We performed a total of 72 tests, e.g. 2 incremental and 4 constant-load tests per patient.
TABLE I.-Characteristics of the study patients.

\begin{tabular}{|c|c|}
\hline Variables & Results \\
\hline Sex, M/F \% & $91.7 / 8.3$ \\
\hline Height $(\mathrm{cm})$ & $168 \pm 7$ \\
\hline Weight (kg) & $75 \pm 13$ \\
\hline $\mathrm{BMI}\left(\mathrm{kg} / \mathrm{m}^{2}\right)$ & $26.7 \pm 6.3$ \\
\hline$\% \mathrm{BMI}<18$ & 16.67 \\
\hline$\% \mathrm{BMI}>18.5<25$ & 8.33 \\
\hline$\% \mathrm{BMI}>25<30$ & 58.33 \\
\hline$\% \mathrm{BMI}>30$ & 16.67 \\
\hline Age (years) & $66 \pm 9$ \\
\hline \multicolumn{2}{|c|}{ Drugs (number [and percentage] of subjects) } \\
\hline - LAMA & $11(92 \%)$ \\
\hline - LABA & $12(100 \%)$ \\
\hline - Inhaled corticosteroids & $11(92 \%)$ \\
\hline - Statins & $3(25 \%)$ \\
\hline - Antiplatelets & $4(33 \%)$ \\
\hline - Antihypertensive & $7(58 \%)$ \\
\hline $\mathrm{FEV}_{1} \%$ pred & $51 \pm 18$ \\
\hline FVC $\%$ pred & $85 \pm 17$ \\
\hline $\mathrm{FEV}_{1} / \mathrm{FVC}$ & $46 \pm 11$ \\
\hline IC \% pred & $90 \pm 12$ \\
\hline RV \% pred & $167 \pm 50$ \\
\hline RV/TLC & $0.54 \pm 0.09$ \\
\hline MIP \% pred & $75 \pm 20$ \\
\hline MEP \% pred & $83 \pm 30$ \\
\hline 6MWT (m) & $504 \pm 63$ \\
\hline $\mathrm{PaO}_{2}(\mathrm{mmHg})$ & $72 \pm 9$ \\
\hline $\mathrm{PaCO}_{2}(\mathrm{mmHg})$ & $39 \pm 2$ \\
\hline $\mathrm{pH}$ & $7.43 \pm 0.03$ \\
\hline $\mathrm{SatO}_{2 \%}$ at rest & $95 \pm 2$ \\
\hline
\end{tabular}

Values expressed as mean \pm DS unless otherwise stated.

BMI: Body Mass Index, LAMA: long-acting antimuscarinic bronchodilators, LABA: long-acting beta-adrenergic bronchodilators, $\mathrm{FEV}_{1}$ : forced expiration volume at 1st second; FVC: forced vital capacity; IC: inspiratory capacity, RV: residual volume; MIP: maximal inspiratory pressure; MEP: maximal expiratory pressure; 6MWT: Six Minute Walk Test; $\mathrm{PaO}_{2}$ : arterial oxygen pressure; $\mathrm{PaCO}_{2}$ arterial $\mathrm{CO}_{2}$ pressure; $\mathrm{SatO}_{2}$ : arterial oxygen saturation.

\section{Preliminary phase}

The mean maximal workload at the end of the incremental supported exercise was $38.75 \pm 10.68$ watts. During the incremental unsupported test (Takahashi Test) the mean maximum weight of the stick lifted was $1.21 \pm 1.08 \mathrm{~kg}$ and the mean maximum level (line reached on the billboard) was $7.42 \pm 0.10$.

\section{Experimental phase}

The median $\mathrm{T}_{\text {lim }}$ in the constant-load tests for supported exercise was 486 (range $330-1140$ ) s at $80^{\circ} v s .360$ (range $210-840)$ second at $120^{\circ}(\mathrm{P}=0.031)$ while for the unsupported exercise $\mathrm{T}_{\lim }$ was 375 (range $240-1200$ ) s at $80^{\circ} v \mathrm{~s}$. 210 (range $120-360) \mathrm{s}$ at $120^{\circ}(\mathrm{P}<0.01)$. Figure 2 shows the Kaplan-Meier curves for $\mathrm{T}_{\text {lim }}$ for each test. A significant 


\section{COPYRIGHT $^{(\odot)} 2018$ EDIZIONI MINERVA MEDICA}

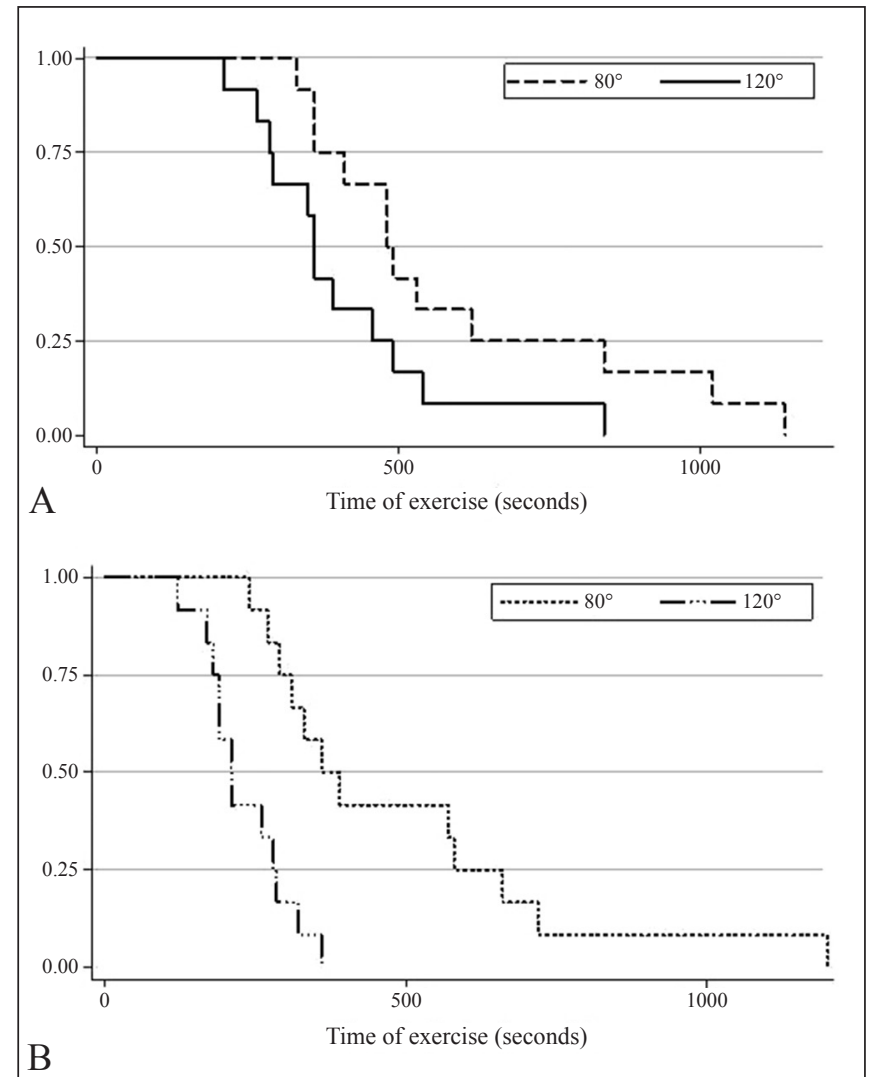

Figure 2.- - Time to exhaustion $\left(\mathrm{T}_{\mathrm{lim}}\right)$ of CL-tests.

Above: supported upper limb exercises. Below: unsupported upper limb exercises.

$80^{\circ}: 80^{\circ}$ degrees of shoulders elevation, $120^{\circ}: 120^{\circ}$ degrees of shoulders elevation.

decrease in $\mathrm{T}_{\text {lim }}$ can be seen in both supported $(\mathrm{P}=0.046)$ and unsupported exercise $(\mathrm{P}<0.01)$ for $120^{\circ}$ elevation with respect to $80^{\circ}$.

\section{Evaluation at peak of exercise}

At peak exercise, no differences between $80^{\circ}$ and $120^{\circ}$ constant-load tests in either the supported and unsupported tests were found in $\mathrm{VO}_{2}$, cardiac (HR), respiratory (RR, $\mathrm{Vt}, \mathrm{VE}$ ) responses or in dyspnea and fatigue symptoms. An increased utilization of the anaerobic metabolic pathways was found only in unsupported exercise at $120^{\circ}$ with respect to $80^{\circ}$, where we found a higher $\mathrm{VCO}_{2}(10.51 \pm 3.23$ vs. $8.78 \pm 2.16, \mathrm{P}<0.05)$ and higher $\mathrm{RQ}(1.09 \pm 0.08$ vs. $0.9766 \pm 0.07, \mathrm{P}<0.01)$. IC decreased significantly during the course of the test in the $80^{\circ}$ supported $(-0.52 \pm 0.39$, $\mathrm{P}<0.01)$ and $120^{\circ}$ unsupported $(-0.16 \pm 0.18, \mathrm{P}<0.02)$ constant-load tests. No significant DH due to exercise was found between groups.

Analysis of each variable normalized to the time to exhaustion

Table II describes the differences of responses in relation to $\mathrm{T}_{\text {lim. }}$. Exercising at $120^{\circ}$ in both supported and unsupported conditions produced a significant increase in respiratory, cardiac and fatigue responses and a major shift from aerobic to anaerobic metabolism, with respect to exercising at $80^{\circ}$ arm elevation. We find a significant difference in the increase of $\mathrm{VO}_{2}, \mathrm{VCO}_{2}$ and perceived dyspnea between $120^{\circ}$ and $80^{\circ}$ elevation only for the unsupported exercise modality.

\section{Analysis at iso-VE}

Iso-VE, defined as the highest common level of $\mathrm{VE}$ reached by all patients in each test, was $20.5 \mathrm{~L} / \mathrm{min}$. At this point of VE, no significant differences were found in any variables or symptoms. IC at iso-VE did not differ

TABLE II.-Metabolic, cardiovascular and respiratory responses to the tests normalized to $T_{\text {lim }}$.

\begin{tabular}{|c|c|c|c|c|c|c|}
\hline Variables & $80^{\circ}$ supported exercise & $\begin{array}{l}120^{\circ} \text { supported } \\
\text { exercise }\end{array}$ & $P$ value & $\begin{array}{l}80^{\circ} \text { unsupported } \\
\text { exercise }\end{array}$ & $\begin{array}{l}120^{\circ} \text { unsupported } \\
\text { exercise }\end{array}$ & $\mathrm{P}$ value \\
\hline VE/time, $\mathrm{mL} / \mathrm{s}$ & $7.06(3.35)$ & $10.59(5.47)$ & 0.041 & $7.45(3.76)$ & $14.99(5.81)$ & $<0.01$ \\
\hline $\mathrm{VO}_{2}$ peak/time, $\mathrm{mL} / \mathrm{s}$ & $1.6(0.78)$ & $3.26(1.44)$ & 0.060 & $1.73(0.74)$ & $2.612(1.44)$ & $<0.01$ \\
\hline $\mathrm{VCO}_{2}$ peak $/$ time $\mathrm{mL} / \mathrm{s}$ & $1.79(7.3)$ & $2.71(1.57)$ & 0.060 & $1.57(7.43)$ & $3.54(1.67)$ & $<0.01$ \\
\hline DeltaIC/time, mL/s & $-0.06(0.05)$ & $-0.04(0.07)$ & 0.182 & $-0.03(0.08)$ & $-0.05(0.056)$ & 0.480 \\
\hline HR/time, beats/s & $0.50(0.17)$ & $0.255(0.1071)$ & $<0.02$ & $0.22(0.85)$ & $0.33(0.12)$ & $<0.01$ \\
\hline Vt/time, mL/s & $2.5(1)$ & $3.7(1.5)$ & 0.034 & $2.9(1.37)$ & $5.3(1.4)$ & $<0.01$ \\
\hline RQ/time, RQ/s & $0.002(0.002)$ & $0.003(0.001)$ & 0.023 & $0.0024(0.0009)$ & $0.0051(0.002)$ & $<0.01$ \\
\hline RR/time, beats/s & $0.63(0.03)$ & $0.09(0.04)$ & $<0.02$ & $0.07(0.03)$ & $0.14(0.06)$ & $<0.01$ \\
\hline Borg dyspnea, score/s & $0.012(0.007)$ & $0.017(0.011)$ & 0.084 & $0.012(0.01)$ & $0.02(0.01)$ & $<0.02$ \\
\hline Borg fatigue, score/s & $0.017(0.006)$ & $0.02(0.008)$ & 0.041 & $0.022(0.009)$ & $0.0413(0.016)$ & $<0.01$ \\
\hline
\end{tabular}

Values expressed as mean \pm SD unless otherwise stated.

VE: minute ventilation, $\mathrm{VO}_{2}$ peak: oxygen uptake at peak of exercise; $\mathrm{VCO}_{2}$ carbon dioxide production at peak of exercise; DeltaIC: difference between basal and postexercise inspiratory capacity; HR: heart rate; Vt: tidal volume; RQ: respiratory quotient $\left(\mathrm{VO}_{2} / \mathrm{VCO}_{2}\right)$; RR: respiratory rate; time: time to exhaustion. 


\section{COPYRIGHT $^{(\odot)} 2018$ EDIZIONI MINERVA MEDICA}

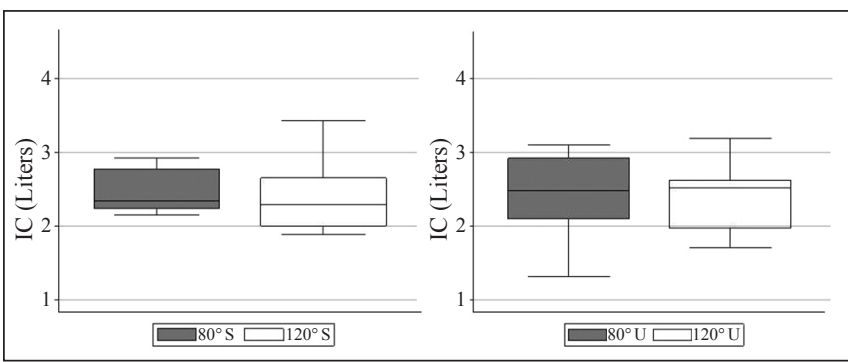

Figure 3.- IC at iso-VE $(20.5 \mathrm{~L} / \mathrm{min})$.

On the left supported upper limb exercises $(\mathrm{P}=0.2550)$ and on the right unsupported upper limb exercises $(\mathrm{P}=0.1167)$.

$\mathrm{S}$ : constant-load supported upper limb exercise; U: constant-load unsupported upper limb exercise; $80^{\circ}: 80^{\circ}$ degrees of shoulders elevation; $120^{\circ}: 120^{\circ}$ degrees of shoulders elevation.

significantly between $120^{\circ}$ and $80^{\circ}$ elevation, as shown in Figure 3. We found a difference in the time required to reach the iso-VE point in supported exercise between $80^{\circ}$ and $120^{\circ}(90.31 \pm 62.80$ vs. 55.22 $\pm 34.81, \mathrm{P}<0.01)$. A trend to significance was detected also for unsupported exercise in the difference between $80^{\circ}$ and $120^{\circ}(128 \pm 127$ vs. 63.67 $\pm 54.48, \mathrm{P}=0.071)$.

\section{Discussion}

Metabolic abnormalities, abnormal gas exchange, abnormal lung mechanics with dynamic hyperinflation, pulmonary arterial hypertension, peripheral artery disease, heart-lung interaction and peripheral muscle dysfunctions are mainly factors limiting exercise in patients affected by COPD. ${ }^{26}$ However, this knowledge has been derived mainly by studies performed by leg exercises and, before now, few studies have shown the pathophysiology responses among different types of arm exercises. Our study helps to better describe this topic.

About the influence of arm elevation on time to exhaustion in 2010, Colucci et al. ${ }^{6}$ showed that the performance during upper limb endurance supported exercise shortened progressively with increasing workloads $(50 \%, 60 \%, 80 \%$ of $\mathrm{VO}_{2 \max }$ loaded by increasing watts on the arm-ergometer). Our results, in relation to their findings, suggest that the increase of shoulder elevation may be considered as a mode to increase the workload applied to arm muscles. ${ }^{8}$

This fact has been described as more evident when unsupported arm exercises which, additionally, resemble more properly the activities of daily living 9 are performed.

In relation to the influence of the degree of arm elevation on DH, Romagnoli et al. ${ }^{27}$ investigated chest wall kinematics by optoelectronic plethysmography during a constant-load unsupported arm exercise at $80 \%$ of peak incremental exercise in COPD patients and suggested that the main cause of interruption of exercise was arm fatigue and not the increase of $\mathrm{DH}$. Our results, describing that arm exercise produced DH irrespective of the height of shoulder flexion, support the mentioned findings.

As regard as the influence of arm elevation on metabolic and ventilatory variables we showed that the arm elevation per se produced an increase of the workload that seems to be greater in unsupported than in supported exercise. Also, during unsupported exercise only, we detected an increased burden of dyspnea that may be related to the more significant shift from aerobic to anaerobic metabolism compared to supported exercise. Our results are in line with those of Dolmage et al., ${ }^{28}$ who investigated the effect of the maintenance of fixed supported and unsupported arm elevation, at rest and during leg exercise, on COPD patients. Albeit in different conditions from ours, unsupported upper limb elevation per se significantly increased the $\mathrm{VO}_{2}, \mathrm{VCO}_{2}, \mathrm{VE}, \mathrm{RR}$ and $\mathrm{Vt}$ with respect to the control position.

Possible explanations for the additional burden caused by shoulder elevation could be: 1) a different pattern of muscle activation. Data on this topic are limited, but Velloso et al. ${ }^{29}$ reported that some domestic activities clearly require a higher proportion of maximal muscle effort (i.e. upper trapezius muscle) in COPD patients with respect to healthy controls; 2) the loss of inspiratory force and the lesser ability to increase ventilation by neck inspiratory muscles, due to the decrease of their length. The findings by Dolmage et al., ${ }^{28}$ demonstrating that arm elevation at rest produced a significant decrease (by $180 \mathrm{~mL}$ ) in vital capacity, supports this suggestion; 3 ) the shift toward anaerobic metabolism while maintaining arm elevation, suggested by the increase in $\mathrm{VCO}_{2}$ and RQ.

A possible reason for the increased anaerobic metabolism utilization could be related to a reduced muscle blood flow delivery to the active muscles, which reduces the local availability of oxygen and a major increase of recruitment of upper back stabilizing muscles that work in prolonged isometric contraction when unsupported exercise is applied.

\section{Clinical implications and further research}

As the inclusion of arm exercise training in pulmonary rehabilitation programs is recommended, ${ }^{6}$ our results highlight the need to consider the position of the shoulders in prescribing the exercise intensity of the arm training. Our findings also suggest that, in arm exercise training, a 


\section{COPYRIGHT $^{(\odot)} 2018$ EDIZIONI MINERVA MEDICA}

gradual and more functional progression of the exercise intensity could be obtained by raising the position of the arm to higher degrees of shoulder flexion, without worsening the DH.

Moreover, although the evaluation of the differences between unsupported and supported exercises was not within the scope of this study, the additional burden in dyspnea found only in unsupported exercise might suggest that one way to progressively increase workload in a rehabilitation program could be through the type of exercise prescribed: starting with supported exercise then increasing the effort by switching to unsupported exercise (which more optimally mimic ordinary tasks). Nevertheless, in this context, the risk of developing shoulder pain, tendinopathies/bursitis, scapular dyskinesia or other conditions would need to be carefully considered, particularly in the elderly or in patients who had previous injuries. Conversely, as activities of the upper limbs are often essential to maintain independence in daily life, a reduction of the burden related to the tasks needs to be considered in some patients. ${ }^{30} \mathrm{Sev}-$ eral energy conservation techniques have been proposed and taught to patients in recent years. ${ }^{31,32}$ Dolmage et al. ${ }^{33}$ showed that, during tasks that included unsupported lifting of the upper limbs, the time to exhaustion can be increased if patients are taught to exhale while raising their arms. In the direction of energy conservation techniques, our data suggest that lowering the height of the shoulders - when performing tasks involving the upper limbs - could reduce the workload and the metabolic cost of the task. Further studies are needed, especially in more severe COPD patients.

This study enables information on arm endurance performance in COPD with a moderate obstruction, mild respiratory muscles impairment, hyperinflation and with a quite preserved exercise tolerance. It is possible that pathophysiological exercise response (i.e. inspiratory rib cage expansion or diaphragmatic and expiratory muscle recruitment) could be different among COPD with different phenotypes, with or without co-morbidities and in patients with diverse obstruction severities.

\section{Limitations of the study}

Our study has some limitations. First, patients were not homogeneous with respect to the level of COPD severity. While all participants were classified as moderateto-severe COPD patients, the SD value of $\mathrm{RV} \%=167 \pm 50$ indicates a moderate variability of resting hyperinflation. We hypothesize that stricter inclusion criteria, e.g. a defined cut-off of RV/TLC or including only patients exhib- iting exercise intolerance due to ventilatory limitations, might lead to different results. Secondly, no evaluation was conducted on the level of muscle activation and on regional blood flow dynamics during the different exercise modalities. On this point, moreover, the experiments were conducted at different times of the day, with possible modifications related to circadian rhythms on many physiological variables such as blood flow control, perceived exertion, maximal attainable force. ${ }^{34}$

\section{Conclusions}

In moderate-to-severe COPD patients, a higher level of shoulder elevation $\left(120^{\circ} v\right.$ s. $\left.80^{\circ}\right)$ during upper limb endurance exercises, both supported and unsupported, worsens per se the performance, elicits a more pronounced metabolic, ventilatory and cardiac response and shifts the metabolism toward the anaerobic pathways without, however, worsening the dynamic hyperinflation. The position of the arms should thus be taken into account when defining exercise training and for educational purposes when teaching patients on how to perform daily life activities optimally.

\section{References}

1. Romer LM, Polkey MI. Exercise-induced respiratory muscle fatigue: implications for performance. J Appl Physiol (1985) 2008;104:879-88. .

2. Leidy NK, Haase JE. Functional status from the patient's perspective: the challenge of preserving personal integrity. Res Nurs Health 1999;22:67-77.

3. Laviolette L, Laveneziana P; ERS Research Seminar Faculty. Dyspnoea: a multidimensional and multidisciplinary approach. Eur Respir J 2014;43:1750-62.

4. Laveneziana P, Palange P; ERS Research Seminar Faculty. Physical activity, nutritional status and systemic inflammation in COPD. Eur Respir J 2012;40:522-9.

5. Laveneziana P, Webb KA, Ora J, Wadell K, O’Donnell DE. Evolution of dyspnea during exercise in chronic obstructive pulmonary disease: impact of critical volume constraints. Am J Respir Crit Care Med 2011;184:1367-73.

6. Colucci M, Cortopassi F, Porto E, Castro A, Colucci E, Iamonti $\mathrm{VC}$, et al. Upper limb exercises using varied workloads and their association with dynamic hyperinflation in patients with COPD. Chest 2010;138:39-46.

7. Ries AL, Bauldoff GS, Carlin BW, Casaburi R, Emery CF, Mahler DA, et al. Pulmonary Rehabilitation: Joint ACCP/AACVPR Evidence-Based Clinical Practice Guidelines. Chest 2007;131(Suppl):4S-42S.

8. Castro AA, Porto EF, Iamonti VC, de Souza GF, Nascimento OA, Jardim JR. Oxygen and ventilatory output during several activities of daily living performed by COPD patients stratified according to disease severity. PLoS One 2013;8:e79727.

9. Garcia-Rio F, Lores V, Mediano O, Rojo B, Hernanz A, López-Collazo $\mathrm{E}$, et al. Daily physical activity in patients with chronic obstructive pulmonary disease is mainly associated with dynamic hyperinflation. Am J Respir Crit Care Med 2009;180:506-12. 


\section{COPYRIGHT $^{(\odot)} 2018$ EDIZIONI MINERVA MEDICA}

10. Casaburi R, ZuWallack R. Pulmonary rehabilitation for management of chronic obstructive pulmonary disease. N Engl J Med 2009;360:1329-35.

11. Porto EF, Castro AA, Nascimento O, Oliveira RC, Cardoso F, Jardim JR. Modulation of operational lung volumes with the use of salbutamo in COPD patients accomplishing upper limbs exercise tests. Respir Med 2009; $103: 251-7$

12. Costi S, Di Bari M, Pillastrini P, D’Amico R, Crisafulli E, Arletti $\mathrm{C}$, et al. Short-term efficacy of upper-extremity exercise training in patients with chronic airway obstruction: a systematic review. Phys Ther $2009 ; 89: 443-55$

13. Martinez FJ, Vogel PD, Dupont DN, Stanopoulos I, Gray A, Beamis JF. Supported arm exercise vs unsupported arm exercise in the rehabilitation of patients with severe chronic airflow obstruction. Chest 1993;103:1397-402.

14. Epstein SK, Celli BR, Martinez FJ, Couser JI, Roa J, Pollock M, et al. Arm training reduces the VO2 and VE cost of unsupported arm exercise and elevation in chronic obstructive pulmonary disease. J Cardiopulm Rehabil 1997;17:171-7.

15. Castro AA, Porto EF, Feltrim MI, Jardim JR. Asynchrony and hyperinflation in patients with chronic obstructive pulmonary disease during two types of upper limbs exercise. Arch Bronconeumol 2013;49:241-8.

16. Couser JI Jr, Martinez FJ, Celli BR. Respiratory response and ventilatory muscle recruitment during arm elevation in normal subjects. Ches 1992;101:336-40.

17. Epstein SK, Celli BR, Williams J, Tarpy S, Roa J, Shannon T. Ventilatory response to arm elevation. Its determinants and use in patients with chronic obstructive pulmonary disease. Am J Respir Crit Care Med 1995;152:211-6.

18. Baarends EM, Schols AM, Slebos DJ, Mostert R, Janssen PP, Wouters EF. Metabolic and ventilatory response pattern to arm elevation in patients with COPD and healthy age-matched subjects. Eur Respir J $1995 ; 8: 1345-51$.

19. Robles P, Araujo T, Brooks D, Zabjek K, Janaudis-Ferreira T, Marzolini $\mathrm{S}$, et al. Does limb partitioning and positioning affect acute cardiorespiratory responses during strength exercises in patients with COPD? Respirology 2017;22:1336-42.

20. Qaseem A, Wilt TJ, Weinberger SE, Hanania NA, Criner G, van der Molen T, et al.; American College of Physicians; American College of Chest Physicians; American Thoracic Society; European Respiratory Society. Diagnosis and management of stable chronic obstructive pulmonary disease: a clinical practice guideline update from the American College of Physicians, American College of Chest Physicians, American
Thoracic Society, and European Respiratory Society. Ann Intern Med 2011;155:179-91.

21. Takahashi T, Jenkins SC, Strauss GR, Watson CP, Lake FR. A new unsupported upper limb exercise test for patients with chronic obstructive pulmonary disease. J Cardiopulm Rehabil 2003;23:430-7.

22. Tanaka H, Monahan KD, Seals DR. Age-predicted maximal heart rate revisited. J Am Coll Cardiol 2001;37:153-6.

23. Borg G. Perceived exertion as an indicator of somatic stress. Scand J Rehabil Med 1970;2:92-8.

24. O’Donnell DE, Revill SM, Webb KA. Dynamic hyperinflation and exercise intolerance in chronic obstructive pulmonary disease. Am J Respir Crit Care Med 2001;164:770-7.

25. Clark TJ, Freedman S, Campbell EJ, Winn RR. The ventilatory capacity of patients with chronic airways obstruction. Clin Sci 1969;36:307-16.

26. Vogiatzis I, Zakynthinos S. The physiological basis of rehabilitation in chronic heart and lung disease. J Appl Physiol (1985) 2013;115:16-21.

27. Romagnoli I, Gigliotti F, Lanini B, Bruni GI, Coli C, Binazzi B, et al. Chest wall kinematics and breathlessness during unsupported arm exercise in COPD patients. Respir Physiol Neurobiol 2011;178:242-9.

28. Dolmage TE, Maestro L, Avendano MA, Goldstein RS. The ventilatory response to arm elevation of patients with chronic obstructive pulmonary disease. Chest 1993;104:1097-100.

29. Velloso M, Stella SG, Cendon S, Silva AC, Jardim JR. Metabolic and ventilatory parameters of four activities of daily living accomplished with arms in COPD patients. Chest 2003;123:1047-53.

30. Meijer K, Annegarn J, Lima Passos V, Savelberg HH, Schols AM, Wouters EF, et al. Characteristics of daily arm activities in patients with COPD. Eur Respir J 2014;43:1631-41.

31. Velloso M, Jardim JR. Functionality of patients with chronic obstructive pulmonary disease: energy conservation techniques. J Bras Pneumol 2006;32:580-6.

32. Silva CS, Nogueira FR, Porto EF, Gazzotti MR, Nascimento OA, Camelier A, et al. Dynamic hyperinflation during activities of daily living in COPD patients. Chron Respir Dis 2015;12:189-96.

33. Dolmage TE, Janaudis-Ferreira T, Hill K, Price S, Brooks D, Goldstein RS. Arm elevation and coordinated breathing strategies in patients with COPD. Chest 2013;144:128-35.

34. Chan-Thim E, Dumont M, Moullec G, Rizk AK, Wardini R, Trutschnigg B, et al. Clinical impact of time of day on acute exercise response in COPD. COPD 2014;11:204-11.

Conflicts of interest.- The authors certify that there is no conflict of interest with any financial organization regarding the material discussed in the manuscript. Authors' contributions. - Mara Paneroni: study design, analysis of data, manuscript preparation, review of manuscript; Carla Simonelli: manuscript preparation and review of the manuscript; Pierantonio Laveneziana: critical revision of manuscript for intellectual content; Massimiliano Gobbo: critical revision of manuscript for intellectual content; Manuela Saleri: data collection and review of the manuscript; Luca Bianchi: data collection, study design and review of the manuscript; Michele Vitacca: study design, manuscript preparation, review of manuscript. All authors approved the final version of the manuscript.

Acknowledegments.-The Authors thank Laura Comini for critical revision and editing the manuscript, and Rosemary Allpress for the English revision of the manuscript.

Article first published online: April 20, 2018. - Manuscript accepted: April 13, 2018. - Manuscript revised: March 22, 2018. - Manuscript received: August 31, 2017. 